\title{
Emerging ultra-narrow-band cyan-emitting phosphor for white LEDs with enhanced color rendition
}

\author{
Ming Zhao', Hongxu Liao', Maxim S. Molokeev²,3, Yayun Zhou', Qinyuan Zhang ${ }^{5}$, Quanlin Liu ${ }^{1}$ and Zhiguo Xia ${ }^{1,5}$
}

\begin{abstract}
Phosphor-converted white LEDs rely on combining a blue-emitting InGaN chip with yellow and red-emitting luminescent materials. The discovery of cyan-emitting $(470-500 \mathrm{~nm})$ phosphors is a challenge to compensate for the spectral gap and produce full-spectrum white light. $\mathrm{Na}_{0.5} \mathrm{~K}_{0.5} \mathrm{Li}_{3} \mathrm{SiO}_{4}: \mathrm{Eu}^{2+}\left(\mathrm{NKLSO}^{2} \mathrm{Eu}^{2+}\right)$ phosphor was developed with impressive properties, providing cyan emission at $486 \mathrm{~nm}$ with a narrow full width at half maximum (FWHM) of only $20.7 \mathrm{~nm}$, and good thermal stability with an integrated emission loss of only $7 \%$ at $150^{\circ} \mathrm{C}$. The ultra-narrow-band cyan emission results from the high-symmetry cation sites, leading to almost ideal cubic coordination for $\mathrm{UCr}_{4} \mathrm{C}_{4}$-type compounds. NKLSO:Eu ${ }^{2+}$ phosphor allows the valley between the blue and yellow emission peaks in the white LED device to be filled, and the color-rendering index can be enhanced from 86 to 95.2, suggesting great applications in full-spectrum white LEDs.
\end{abstract}

\section{Introduction}

White light-emitting diodes (LEDs) have been successfully used in solid-state lighting or backlight units for liquid crystal displays due to their high efficiency, tunable color, durability, long lifetime, energy saving, and environmental friendliness ${ }^{1-3}$. Currently, most commercial phosphor-converted LEDs ( $p c$-LEDs) can be achieved by the combination of a blue InGaN chip with yellowemitting $\mathrm{Y}_{3} \mathrm{Al}_{5} \mathrm{O}_{12}: \mathrm{Ce}^{3+}$ (YAG:Ce) phosphor ${ }^{4,5}$. It is acknowledged that such white LEDs have some drawbacks, such as a low color-rendering index (CRI, $R_{\mathrm{a}}<80$ ) and high correlated color temperature (CCT) (CCT > $4000 \mathrm{~K}$ ), owing to the lack of the red region of the spectrum $^{6}$. After the discovery of highly efficient red-emitting phosphors, such as CaAlSiN $3: \mathrm{Eu}^{2+}, \mathrm{Sr}_{2} \mathrm{Si}_{5} \mathrm{~N}_{8}: \mathrm{Eu}^{2+}, \mathrm{K}_{2} \mathrm{SiF}_{6}$ : $\mathrm{Mn}^{4+}$, or $\operatorname{SrLiAl}_{3} \mathrm{~N}_{4}: \mathrm{Eu}^{2+}$, the $R_{\mathrm{a}}$ values can be enhanced

\footnotetext{
Correspondence: Zhiguo Xia (xiazg@ustb.edu.cn)

${ }^{1}$ The Beijing Municipal Key Laboratory of New Energy Materials and

Technologies, School of Materials Sciences and Engineering, University of Science and Technology Beijing, 100083 Beijing, China

${ }^{2}$ Laboratory of Crystal Physics, Kirensky Institute of Physics, Federal Research Center KSC SB RAS, Krasnoyarsk, 660036, Russia

Full list of author information is available at the end of the article.
}

to $\sim 90^{7-11}$. Moreover, some solid solution phosphors with multiple emission centers have been discovered to improve the $R_{\mathrm{a}}$ values $^{11,12}$. However, there is still a challenge in enhanced color rendition because of the cyan gap between blue and yellow emission in the $470-500 \mathrm{~nm}$ region, which is not suitable for high-quality general lighting. Hence, it is of fundamental importance to develop a novel phosphor emitting in this spectral region for filling the valley between the blue and yellow emission peaks in a white LED device. Accordingly, the development of narrow-band cyan-emitting phosphors excited by blue light with a small Stokes shift is necessary for improving the optical performance of pc-LEDs ${ }^{13}$.

The design of narrow-band cyan-emitting phosphors plays a significant role in enhancing the color rendition to compensate for the peak valley between the blue and yellow emission peaks. However, luminous efficacy and color rendition are generally in a trade-off relationship $^{14,15}$. Thus, the narrow-band characteristic will decrease the opportunity for spectral overlapping of the excitation spectrum of YAG:Ce and emission spectrum of the cyan-emitting phosphor, which avoids the

\section{(c) The Author(s) 2019}

(c) (i) Open Access This article is licensed under a Creative Commons Attribution 4.0 International License, which permits use, sharing, adaptation, distribution and reproduction c. in any medium or format, as long as you give appropriate credit to the original author(s) and the source, provide a link to the Creative Commons license, and indicate if changes were made. The images or other third party material in this article are included in the article's Creative Commons license, unless indicated otherwise in a credit line to the material. If material is not included in the article's Creative Commons license and your intended use is not permitted by statutory regulation or exceeds the permitted use, you will need to obtain permission directly from the copyright holder. To view a copy of this license, visit http://creativecommons.org/licenses/by/4.0/. 
reabsorption effect to realize the maximum achievable luminous efficacy ${ }^{4}$. Presently, there are few narrow-band cyan-emitting phosphors that can be excited by blue light. The typical example is $\mathrm{BaSi}_{2} \mathrm{O}_{2} \mathrm{~N}_{2}: \mathrm{Eu}^{2+}$, which possesses an emission band with the peak at $\sim 495 \mathrm{~nm}$ (FWHM $=$ $\sim 32 \mathrm{~nm})^{16}$. However, it has poor chemical and thermal stability due to its layered crystal structure. Recently, a new narrow-band cyan-emitting oxonitridoberyllate phosphor $\mathrm{Sr}\left[\mathrm{Be}_{6} \mathrm{ON}_{4}\right]$ : $\mathrm{Eu}^{2+}\left(\lambda_{\mathrm{em}}=495 \mathrm{~nm}, \mathrm{FWHM}=35\right.$ $\mathrm{nm})$ has been reported ${ }^{13}$. Nevertheless, the harsh synthetic conditions and toxicity of this phosphor are serious drawbacks for its application. Therefore, novel stable and nontoxic phosphors with narrow-band cyan emission are extremely required for application in enhancing the color rendition of $p c$-LEDs.

Recently, the development of narrow-emitting phosphors has been continuously pursued for versatile applications in solid-state lighting or backlight units for liquid crystal displays, and understanding structure-property relations for the creation of narrow-band emission is also a great challenge ${ }^{17}$. The mineral-inspired prototype evolution and new phase construction proposed by our group have demonstrated great potential in discovering new phosphors for emerging applications ${ }^{5,18}$. For example, the newly reported narrow-band materials, Be-containing phosphors, $\mathrm{SrLi}_{2}\left[\mathrm{Be}_{4} \mathrm{O}_{6}\right]$ is related to the $\mathrm{BaLi}_{2}\left[\left(\mathrm{Al}_{2} \mathrm{Si}_{2}\right)\right.$ $\left.\mathrm{N}_{6}\right]$ prototype, and $\mathrm{Sr}\left[\mathrm{BeSi}_{2} \mathrm{~N}_{4}\right]$ originates from the $\mathrm{Sr}$ $\left[\mathrm{Be}_{3} \mathrm{O}_{4}\right]$ model $^{19-21}$. Moreover, narrow-band nitride phosphors are extensively investigated in the $\mathrm{UCr}_{4} \mathrm{C}_{4}$-type model, and one of the typical examples is red-emitting $\mathrm{Sr}$ $\left[\mathrm{LiAl}_{3} \mathrm{~N}_{4}\right]: \mathrm{Eu}^{2+}$ with a highly condensed, rigid network, and highly symmetric dopant sites ${ }^{10}$. Therefore, oxidebased $\mathrm{UCr}_{4} \mathrm{C}_{4}$-type compounds with narrow-band emission can also be expected ${ }^{22-24}$. Inspired by these findings, an emerging ultra-narrow-band cyan-emitting phosphor in silicate materials was demonstrated in this work, and we adopted structural modeling in this system to design a novel narrow-band cyan-emitting silicate phosphor. Accordingly, the totally new $\left[\mathrm{Na}_{0.5} \mathrm{~K}_{0.5}\right]\left[\mathrm{Li}_{3} \mathrm{Si}_{\mathrm{O}} \mathrm{O}_{4}\right.$ phase, originating from the $\mathrm{UCr}_{4} \mathrm{C}_{4}$-type compound $\mathrm{NaLi}_{3} \mathrm{SiO}_{4}$, was discovered in this host family. The relationship between the ultra-narrow-band cyan emission and three different cations with high-symmetry cation sites leading to almost ideal cubic coordination was analyzed. $\mathrm{Eu}^{2+}$ doped $\left[\mathrm{Na}_{0.5} \mathrm{~K}_{0.5}\right]\left[\mathrm{Li}_{3} \mathrm{Si}\right] \mathrm{O}_{4}$ (abbreviated as NKLSO:Eu ${ }^{2+}$ ) phosphor exhibits an ultra-narrow-band emission with a peak at $486 \mathrm{~nm}$, FWHM $=20.7 \mathrm{~nm}$ and a Stokes shift $=$ $1069 \mathrm{~cm}^{-1}$. Compared to that of the red-emitting $\mathrm{Sr}$ $\left[\mathrm{LiAl}_{3} \mathrm{~N}_{4}\right]: \mathrm{Eu}^{2+}$ phosphor, the emission peak of NKLSO: $\mathrm{Eu}^{2+}$ has a significant blueshift. Because the formation energy of $\mathrm{N}^{3-}$ from atomic $\mathrm{N}\left(+2300 \mathrm{~kJ} \mathrm{~mol}^{-1}\right)$ is higher than that of $\mathrm{O}^{2-}$ from atomic $\mathrm{O}\left(+700 \mathrm{~kJ} \mathrm{~mol}^{-1}\right)$, the bonding in nitrides is notably more covalent than that in oxides $^{25}$. Therefore, the $5 d$ energy levels of $\mathrm{Eu}^{2+}$ in nitride would be lower compared to that in oxide, which results in the lower $5 d-4 f$ transition energy of the $\operatorname{Sr}\left[\mathrm{LiAl}_{3} \mathrm{~N}_{4}\right]$ : $\mathrm{Eu}^{2+}$ nitride phosphor than that of the NKLSO: $\mathrm{Eu}^{2+}$ oxide phosphor. Moreover, this NKLSO:Eu ${ }^{2+}$ phosphor possesses good thermal stability with an integrated emission intensity of $93 \%$ at $150{ }^{\circ} \mathrm{C}$. By combining the cyan phosphor NKLSO:8\% $\mathrm{Eu}^{2+}$, the commercial yellow phosphor YAG:Ce and the commercial red phosphor $\mathrm{K}_{2} \mathrm{SiF}_{6}: \mathrm{Mn}^{4+}\left(\mathrm{KSF}: \mathrm{Mn}^{4+}\right)$ with a blue LED chip, a white LED with a high $R_{\mathrm{a}}$ of 95.2 was acquired, which demonstrated that the phosphor can cover the cyan gap to enhance the CRI in the as-fabricated white LED.

\section{Results}

\section{Crystal structure and morphology of NKLSO}

Figure 1a shows the XRD patterns of NKLSO and NKLSO: $8 \% \mathrm{Eu}^{2+}$, and all the diffraction peaks can be indexed to the reported pattern of $\mathrm{RbNa}_{3} \mathrm{Li}_{8}\left(\mathrm{Li}\left(\mathrm{SiO}_{4}\right)\right)_{4}$ (PDF card No.82-0818) ${ }^{26}$, which indicates that NKLSO is isostructural to it. Hence, the crystal structure of $\mathrm{RbNa}_{3}$ $\mathrm{Li}_{8}\left(\mathrm{Li}_{(}\left(\mathrm{SiO}_{4}\right)\right)_{4}{ }^{26}$ is taken as the starting model for the Rietveld refinement of NKLSO. Three cation sites (Rb, $\mathrm{Na} 1$, and $\mathrm{Na} 2$ ) exist in the host, and all of them are occupied by $\mathrm{K} / \mathrm{Na}$ mixed ions. The ratios are allowed to refine with restriction that the sum of occupations occ $(\mathrm{Na})+\operatorname{occ}(\mathrm{K})$ is equal to 1 at all sites. After preliminary refinement, it is found that the first cation is fully occupied by $\mathrm{K}^{+}$ions, the second site is intermixed $\mathrm{K} / \mathrm{Na}$ with a ratio of $0.67 / 0.33$, and the third site is fully occupied by $\mathrm{Na}^{+}$ ions (Fig. 1b). These occupations were accounted for in the new model, and $\mathrm{K} / \mathrm{Na}$ ratios were fixed in the first and third sites. The thermal parameters of all ions were refined isotropically. The as-obtained structural parameters were stable with low $R$-factors (Supplementary Fig. S1a and Supplementary Table S1). NKLSO was found to crystallize in a tetragonal crystal system with space group $I 4 / m$, and its unit cell parameters are $a=b=10.9447$ (1) $\AA$ and $c=$ 6.26244 (8) A. Coordinates of atoms and main bond lengths are shown in Supplementary Table S2 and Supplementary Table S3, respectively. The chemical formula from refinement can be written as $\mathrm{Na}_{0.58(4)} \mathrm{K}_{0.42(4)} \mathrm{Li}_{3} \mathrm{SiO}_{4}$, which is close to the suggested formula within $2 \sigma$. The crystallographic information file of NKLSO is also provided in the Supplementary Information.

Regarding the crystal structure (Fig. 1b and Supplementary Fig. S1b), $\mathrm{LiO}_{4}$ and $\mathrm{SiO}_{4}$ tetrahedra connected to each other by corner- and edge-sharing to form a highly condensed three-dimensional framework with the degree of condensation $\kappa=1$ that is equal to the atomic ratio $(\mathrm{Li}$, $\mathrm{Si}$ ): $\mathrm{O}$, and three different cations, $\mathrm{K} 1, \mathrm{~K} 2 / \mathrm{Na} 2$, and $\mathrm{Na}$, are filled in vierer ring channels along [001]. All of these ions are coordinated with eight $\mathrm{O}^{2-}$ ions forming cubic polyhedrons with high-symmetry characteristics (Fig. 1b). For example, the high-symmetry $4 / m$ of the $K 1$ and 


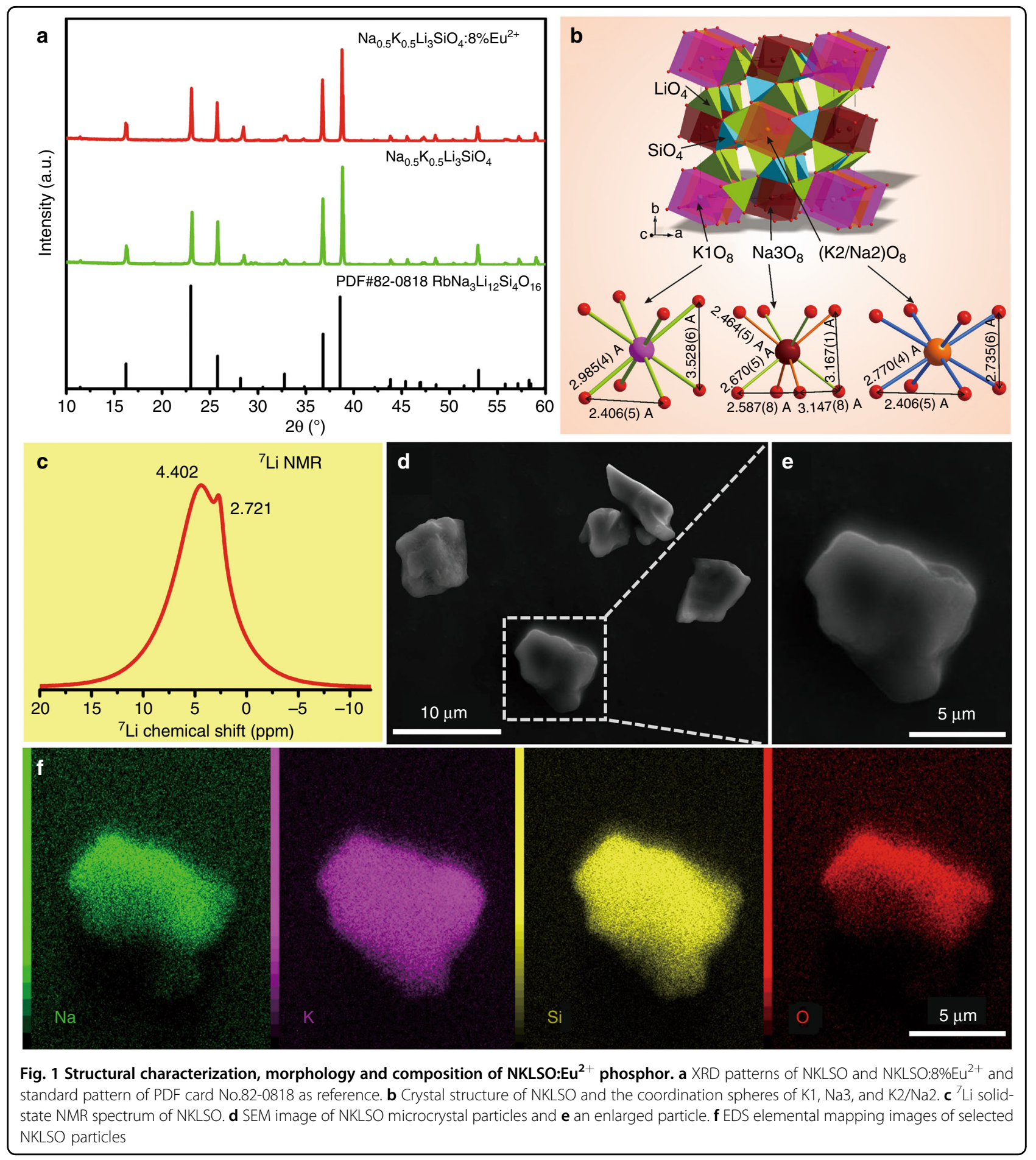

$\mathrm{K} 2 / \mathrm{Na} 2$ sites lead to almost ideal cubic coordination with equal $d_{(\mathrm{Na}, \mathrm{K}-\mathrm{O})}$ bond lengths and with a difference between some $d_{(\mathrm{O}-\mathrm{O})}$ only (Fig. 1b), which strongly contribute to the as-observed narrow emission bands, as discussed below. The $\mathrm{Na} 3$ site has a relatively low symmetry of -4 , and the bond lengths $d_{(\mathrm{Na} 3-\mathrm{O})}$ divide into two groups, leading to distorted cubic coordination. The alkali metal ions replaced by $\mathrm{Eu}^{2+}$ would lead to an increase in positive charge of the unit cell; hence, the charge compensation can be associated with the formation of vacancies in cations instead of anions, such as $2 \mathrm{Na}^{+} \rightarrow \mathrm{Eu}^{2+}+\mathrm{V}_{\mathrm{Na}}$, $2 \mathrm{~K}^{+} \rightarrow \mathrm{Eu}^{2+}+\mathrm{V}_{\mathrm{K}}, \mathrm{Li}^{+}+\mathrm{K}^{+} \rightarrow \mathrm{Eu}^{2+}+\mathrm{V}_{\mathrm{Li}}$, and $\mathrm{Li}^{+}+$ $\mathrm{Na}^{+} \rightarrow \mathrm{Eu}^{2+}+\mathrm{V}_{\mathrm{Li}}$. The numerous different combinations prevent reliable determination of the mechanism, and 
therefore, we do not speculate about it. However, these substitutions and as-formed vacancies will not have a strong influence on the $\mathrm{Eu}^{2+}$ cuboid-like coordination with the relatively stable first coordination sphere of $\mathrm{O}^{2-}$ ions.

The local structure, chemical composition and morphology of NKLSO: $\mathrm{Eu}^{2+}$ phosphor were further investigated to assess this newly discovered ultra-narrow-band cyan-emitting phosphor. First, the ${ }^{7} \mathrm{Li}$ solid-state nuclear magnetic resonance (NMR) spectrum was measured. As displayed in Fig. 1c, the ${ }^{7} \mathrm{Li}$ NMR spectrum shows two signals at 2.721 and $4.402 \mathrm{ppm}$, which are consistent with the two different Li crystallographic sites in this host. Scanning electron microscope (SEM) images of the NKLSO powder sample are depicted in Fig. 1d, e, which indicates that the average particle size of the sample is $\sim 5-10 \mu \mathrm{m}$, and a well-developed crystalline particle and smooth surface will help the luminescence properties and incorporation into LED packages. The elemental mapping images (Fig. 1f) show a uniform distribution of $\mathrm{Na}, \mathrm{K}, \mathrm{Si}$, and $\mathrm{O}$ in the particle, and the average atomic ratios $\mathrm{Na}$ (0.5): $\mathrm{K}(0.44): \mathrm{Si}(1.1)$ determined by energy dispersive $\mathrm{X}$ ray spectroscopy (EDS) are consistent with the formula $\mathrm{Na}_{0.58(4)} \mathrm{K}_{0.42(4)} \mathrm{Li}_{3} \mathrm{SiO}_{4}$ obtained from the Rietveld refinement result from the XRD pattern.

\section{Photoluminescence properties}

Figure 2a gives the photoluminescence (PL) spectra of NKLSO: $x \mathrm{Eu}^{2+}(x=1-15 \%)$ at room temperature (RT), and the optimum $\mathrm{Eu}^{2+}$ concentration is determined to be $8 \mathrm{~mol} \%$. The diffuse reflectance spectra of NKLSO: $x \mathrm{Eu}^{2+}$ phosphors are demonstrated in Supplementary Fig. S2, and the absorption intensities increase gradually with increasing $\mathrm{Eu}^{2+}$ concentration. Fig. $2 \mathrm{~b}$ shows the diffuse reflectance, photoluminescence excitation (PLE) and PL spectra at RT of NKLSO:8\%Eu ${ }^{2+}$ phosphor. The PLE spectra monitored at two different wavelengths, 486 and $510 \mathrm{~nm}$, show a broad band from 300 to $500 \mathrm{~nm}$, indicating that the phosphor can be excited by ultraviolet light to blue light and is suitable for excitation of the commercial blue chip. The diffuse reflectance spectrum gives a broad absorption band in the range of $330-470 \mathrm{~nm}$, which matched well with the PLE spectrum. The PL spectrum of the NKLSO:8\%Eu ${ }^{2+}$ phosphor consists of a dominant asymmetric narrow-band peak at $486 \mathrm{~nm}$ $(\mathrm{FWHM}=20 \mathrm{~nm})$ and a subordinate shoulder peak at $530 \mathrm{~nm}$ under $400 \mathrm{~nm}$ excitation, indicating that other luminescence centers may exist, which will be discussed later. In addition, we measured the PL spectra of NKLSO: $8 \% \mathrm{Eu}^{2+}$ under different excitation wavelengths. As shown in Supplementary Fig. S3a, compared to that of the dominant peak at $486 \mathrm{~nm}$, the intensity of the minor peak at $530 \mathrm{~nm}$ increased obviously with increasing excitation wavelength, which further proves the existence of other luminescence centers and different evolution behaviors. We calculated the Commission Internationale de L'Eclairage (CIE) chromaticity coordinates and the color purity of the PL spectra of NKLSO: $8 \% \mathrm{Eu}^{2+}$ under $400 \mathrm{~nm}$ and $460 \mathrm{~nm}$ excitation. The CIE chromaticity coordinates were calculated to be $(0.1237,0.4098)$ and $(0.1430,0.4380)$, respectively (Supplementary Fig. S3b), and the color purities were determined to be $68.7 \%$ and $66.8 \%$. Under the excitation of $400 \mathrm{~nm}$ and $460 \mathrm{~nm}$, the color coordinates of the phosphor moved slightly but still exhibited cyan emission, and the color purities remained almost unchanged. Moreover, the FWHM values of NKLSO: $x \mathrm{Eu}^{2+}$ remained basically unchanged for different $\mathrm{Eu}^{2+}$ doping concentrations (Fig. 2a). The inset image in Fig. $2 b$ shows that the sample has cyan light under $365 \mathrm{~nm}$ lamp irradiation. The highly condensed and rigid network results in the narrow-band emission and the small Stokes shift, which is determined as the energy difference between the maximum of the (lowest) excitation band and that of the emission band ${ }^{27}$. The extremely small Stokes shift $\left(1069 \mathrm{~cm}^{-1}\right)$ of NKLSO:8\% $\mathrm{Eu}^{2+}$ also accounts for the confinement in the local structure relaxation of $\mathrm{Eu}^{2+}$ in its excited state. Under $400 \mathrm{~nm}$ excitation, the RT internal/ external quantum efficiencies of $\mathrm{NKLSO}: 8 \% \mathrm{Eu}^{2+}$ are determined to be $76 \% / 30 \%$, respectively, and the measurement details are shown in Supplementary Fig. S4.

To further verify the correlation between the existing luminescent centers and the crystallographic sites in the crystal structure, the PL spectrum of NKLSO:8\% $\mathrm{Eu}^{2+}$ at $80 \mathrm{~K}$ was measured. It is commonly known that fine emission spectra can be observed at low temperatures owing to decreased thermal broadening. As shown in Fig. 2c, three emission bands located at $486 \mathrm{~nm}, 495 \mathrm{~nm}$, and $529 \mathrm{~nm}$ are clearly observed, which can be ascribed to the existence of three luminescence centers originating from three cation sites occupied by $\mathrm{Eu}^{2+}$, as elucidated in the crystal structure analysis. Furthermore, the PL spectrum at $80 \mathrm{~K}$ can be divided into three Gaussian peaks (Fig. 2c). Two narrow peaks with FWHM 4.7/14.3 nm can be associated with the high cubic symmetry sites, K1 and $\mathrm{K} 2 / \mathrm{Na} 2$ sites occupied by $\mathrm{Eu}^{2+}$, and the slightly broad emission band with FWHM $51.8 \mathrm{~nm}$ can be associated with $\mathrm{Na} 3$ sites occupied by $\mathrm{Eu}^{2+}$ owing to the distorted cubic sites. Since we could not refine Eu occupancies in different cation sites using Rietveld refinement due to the very low $\mathrm{Eu}$ concentration, the well-known empirical equation given by Van Uitert can be used to further understand the origin of the three emission bands, which provides a good fit to the emission peak for $\mathrm{Eu}^{2+}$ discussed hereafter ${ }^{28}$.

$$
E=Q\left[1-\left(\frac{V}{4}\right)^{\frac{1}{V}} 10^{-\frac{n \times \mathrm{EA} \times r}{80}}\right]
$$



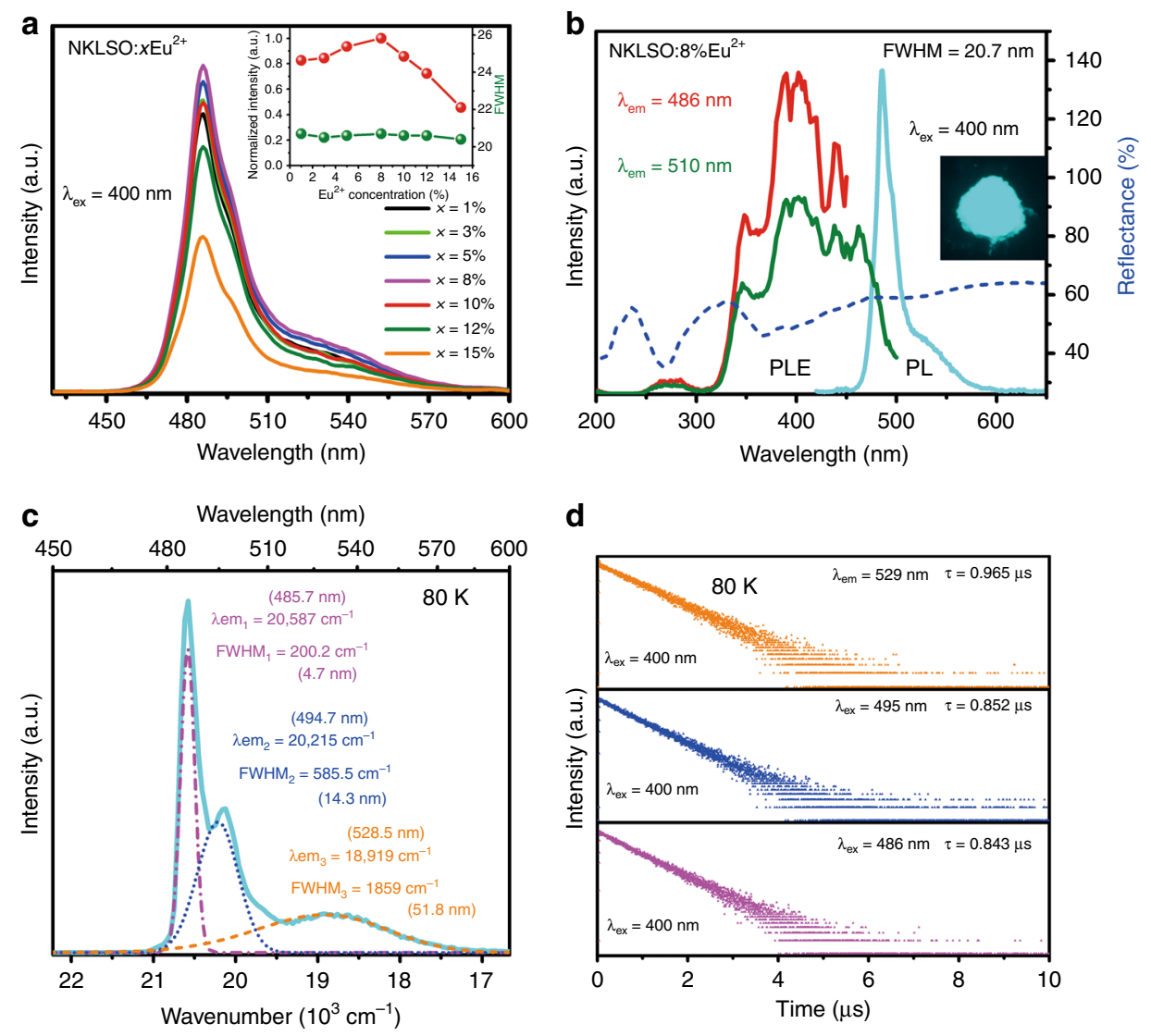

d

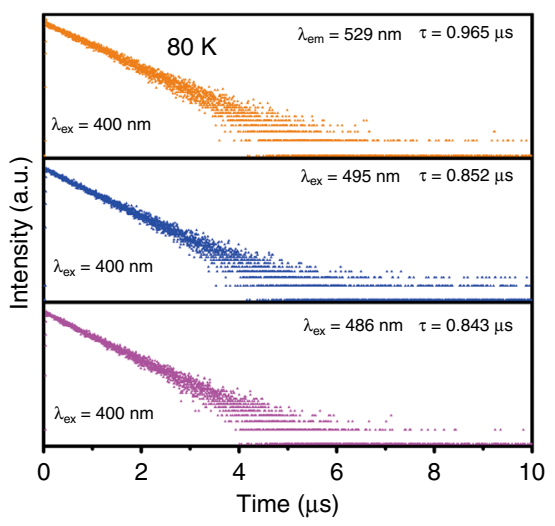

Fig. 2 Photoluminescence properties of NKLSO:Eu ${ }^{2+}$. a Emission spectra of NKLSO:xEu ${ }^{2+}(x=1-15 \%)$. The inset shows the dependence of normalized integrated emission intensities and FWHM values on the Eu ${ }^{2+}$ doping concentration. $\mathbf{b}$ Diffuse reflectance, photoluminescence and photoluminescence excitation spectra of $\mathrm{NKLSO}: 8 \% \mathrm{Eu}^{2+}$ at room temperature. The inset shows a digital photograph of the cyan $\mathrm{NKLSO} 8 \% \mathrm{Eu}^{2+}$ phosphor under a $365 \mathrm{~nm}$ UV lamp. c Emission spectrum (cyan line) of NKLSO:8\%Eu ${ }^{2+}$ at $80 \mathrm{~K}$, and the Gaussian peaks fitting the emission spectrum

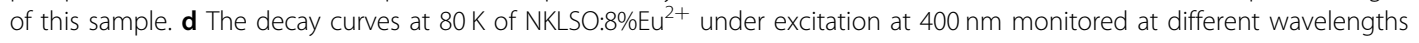

where $E$ denotes the position of the $d$-band edge in energy $\left(\mathrm{cm}^{-1}\right), Q$ represents the position in energy for the lower $d$-band edge of the free ions, $Q$ is $34,000 \mathrm{~cm}-1$ for $\mathrm{Eu}^{2+}$ and $50,000 \mathrm{~cm}^{-1}$ for $\mathrm{Ce}^{3+}, V$ is the valence of the active ion (here, $V=2), n$ is the number of anions in the immediate shell around, $E A$ is the electron affinity of the atoms that form anions, and $r$ is the radius of the host cation replaced by the active cation. Here, $n=8$ for the $\mathrm{K} 1, \mathrm{~K} 2 / \mathrm{Na} 2$, and $\mathrm{Na} 3$ sites, $E A$ should have the same values for $\mathrm{K} 1, \mathrm{~K} 2 / \mathrm{Na} 2$, and $\mathrm{Na} 3$ in this host, and $r(\mathrm{~K} 1)>r$ $(\mathrm{K} 2 / \mathrm{Na} 2)>r(\mathrm{Na} 3)$. From the equation, we can see that the larger the value of $r$ is, the larger the value of $E$, and the smaller the emission wavelength. Hence, the first peak $(486 \mathrm{~nm})$ in the PL spectrum can be assigned to K1 sites, the second peak $(495 \mathrm{~nm})$ can be assigned to K2/ $\mathrm{Na} 2$ sites, and the third broad peak $(529 \mathrm{~nm})$ can be assigned to $\mathrm{Na} 3$ sites, which is consistent with the previous analysis results depending on different symmetries. Furthermore, the decay behaviors of $\mathrm{Eu}^{2+}$ at various sites are generally different depending on the variable chemical environment. To further verify the existence of three $\mathrm{Eu}^{2+}$ emissions in NKLSO:Eu ${ }^{2+}$, the decay curves at $80 \mathrm{~K}$ monitored at 486, 495, and $529 \mathrm{~nm}$ under $400 \mathrm{~nm}$ excitation are measured (Fig. 2d). The decay times are determined to be $0.843,0.852$, and $0.965 \mu$ s for the peaks at 486,495 , and $529 \mathrm{~nm}$, respectively. The different lifetimes demonstrated that the emissions arise from $\mathrm{Eu}^{2+}$ in different lattice sites, and the close decay times $(0.843$ and $0.852 \mu \mathrm{s})$ are ascribed to the quite similar $4 / m$ sites (K1, $\mathrm{K} 2 / \mathrm{Na}$ ) in the lattice, while the value of $0.965 \mu$ s should be ascribed to $\mathrm{Eu}^{2+}$ at the different $\mathrm{Na} 3$ sites with a relatively low symmetry of -4 .

\section{Thermal quenching properties}

Thermal stability acts as a vital parameter for LED phosphors in practical applications since the LED chip normally reaches temperatures up to $\sim 150{ }^{\circ} \mathrm{C}$ at high power. The thermal quenching behavior can be elucidated using the configurational coordinate diagram. Based on this model, the excited luminescent center is thermally 

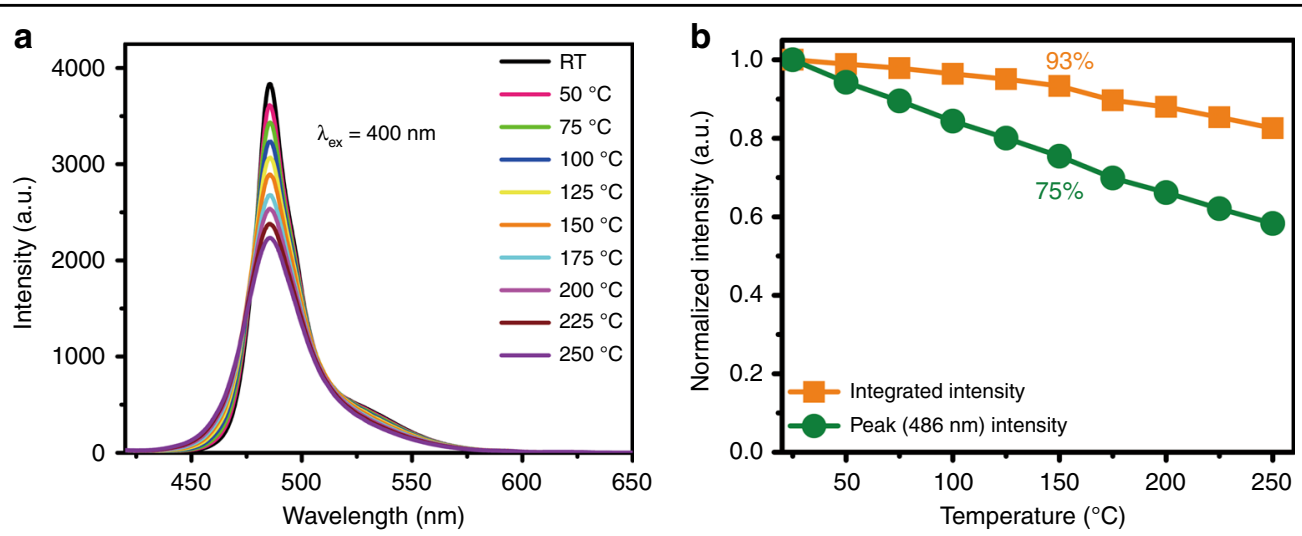

Fig. 3 Thermal quenching behavior of NKLSO:8\%Eu ${ }^{2+}$ phosphor. a Temperature-dependent emission spectra of NKLSO:8\%Eu ${ }^{2+}$ phosphor under $400 \mathrm{~nm}$ excitation in the temperature range $\mathrm{RT}-250^{\circ} \mathrm{C}$ with a temperature interval of $25^{\circ} \mathrm{C}$. $\mathbf{b}$ Temperature-dependent normalized integrated emission intensities and normalized peak $(486 \mathrm{~nm})$ intensities of NKLSO:8\%Eu ${ }^{2+}$

activated through phonon interaction and then released through the crossing point between the excited and ground states ${ }^{29}$. Hence, thermal quenching is related to the difference between the equilibrium distance of the ground and excited state $(\Delta \mathrm{R})$ in the configurational coordinate diagram, which determines the location of the crossing point ${ }^{30}$. Thus, if the value of $\Delta \mathrm{R}$ is small, the Stokes shift will be small ${ }^{27}$. Generally, a phosphor with a smaller Stokes shift has stronger structural rigidity ${ }^{31}$, which could significantly reduce the emission loss with increasing temperature. The temperature-dependent emission spectra of NKLSO: $8 \% \mathrm{Eu}^{2+}$ phosphor in the temperature range from $\mathrm{RT}$ to $250^{\circ} \mathrm{C}$ are shown in Fig. 3a. At $150^{\circ} \mathrm{C}$, NKLSO: $8 \% \mathrm{Eu}^{2+}$ exhibits an integrated emission loss of only $7 \%$, and the emission intensity of the peak $(486 \mathrm{~nm})$ is decreased by $25 \%$ of the initial intensity, indicating good thermal stability of NKLSO:Eu ${ }^{2+}$ (Fig. 3b). And the variation between the peak intensity and the integrated intensity can be attributed to the broadening effect of the emission band with increasing temperature.

\section{Performance of as-fabricated white LEDs for enhancing color rendition}

To further evaluate the potential application of NKLSO: $\mathrm{Eu}^{2+}$ in white LED lighting for improving the colorrendering index, we fabricated white LED lamps by using the cyan phosphor NKLSO:8\%Eu ${ }^{2+}$, the commercial yellow phosphor YAG:Ce and the commercial red phosphor KSF: $\mathrm{Mn}^{4+}$ on a blue LED InGaN chip $(\lambda=455 \mathrm{~nm})$ under various drive currents and compared them with the white LED without the cyan phosphor NKLSO:Eu ${ }^{2+}$. Figure 4a, b comparatively show the emission spectra of the asfabricated LED1 (without the sample) and LED2 (with the sample) devices under a current of $20 \mathrm{~mA}$, and the insets show the photographs of the fabricated and lit white LEDs. LED1 presents warm white light with a CCT of
$4119 \mathrm{~K}$, a $R_{\mathrm{a}}$ of 86 and chromaticity coordinates of (0.3742, 0.3690). However, LED1 shows a cyan gap causing the limited enhancement in the color-rendering index $\left(R_{\mathrm{a}}\right)$, which is difficult to exceed 90. As the cyan phosphor NKLSO:8\% $\mathrm{Eu}^{2+}$ was added into the device, LED2 shows a similar CCT of $4021 \mathrm{~K}$ and chromaticity coordinates of $(0.3835,0.3910)$, but the $R_{\mathrm{a}}$ can be enhanced to 95.2, indicating the great application potential of the device in full-spectrum lighting for enhanced color rendition. The emission spectra and the variation in chromaticity coordinates of the white LED devices under various drive currents are shown in Supplementary Fig. S5, Supplementary Table S4, and Supplementary Table S5. The provided photoelectric parameters also show that the chromaticity coordinates of LED1 shift more than those of LED2, indicating that LED2 has better color stability when the drive current increases from 20 to $120 \mathrm{~mA}$.

\section{Discussion}

The comparison of the emission spectra of LED1 and LED2 (Fig. 4c) reveals three main features: (1) an additional peak appears at $\sim 490 \mathrm{~nm}$ in the spectrum of LED2, and it truly compensates for the cyan gap between $470 \mathrm{~nm}$ and $500 \mathrm{~nm}$; (2) this cyan-emitting spectrum shows a decrease in the intensity of the two peaks at $455 \mathrm{~nm}$ and $550 \mathrm{~nm}$, indicating the noticeable increase in absorption of the blue part in the range of $380-600 \mathrm{~nm}$; and (3) narrow high-intensity peaks in the range of $600-650 \mathrm{~nm}$ stay almost unchanged, causing the relative intensity of the red part to increase compared with that of the blue part. The spectral evolution characteristics are complex, and then it was decided to calculate the difference between the spectra to predict the theoretical maximum of $R_{\mathrm{a}}$. Herein, we define the following relationship: DIFF $(\lambda)=\operatorname{LED} 2(\lambda)-\operatorname{LED} 1(\lambda)$, where $\operatorname{LED} 2(\lambda)$ is the experimental spectrum of LED2 and $\operatorname{LED} 1(\lambda)$ is the 


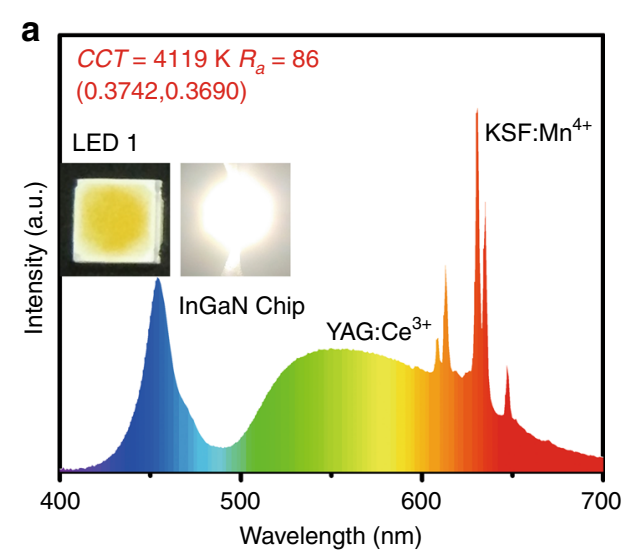

b
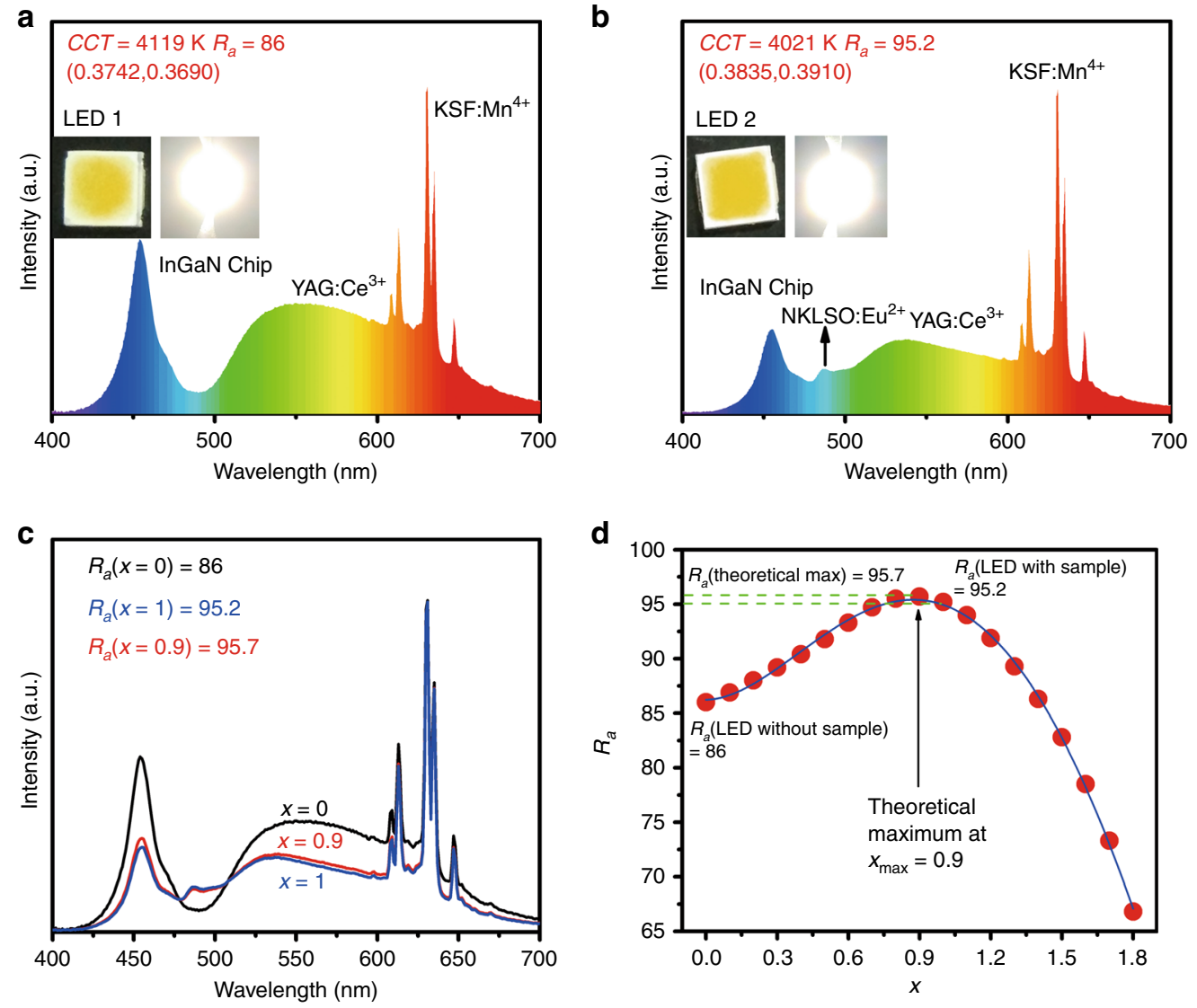

Fig. 4 Performance of as-fabricated white LED devices. Emission spectra of the white LED devices fabricated with the commercial yellow phosphor YAG:Ce, the commercial red phosphor KSF:Mn ${ }^{4+}$ and a without or $\mathbf{b}$ with the cyan phosphor NKLSO:8\%Eu ${ }^{2+}$ on a blue LED InGaN chip $(\lambda=455 \mathrm{~nm})$ under a current of $20 \mathrm{~mA}$. The insets show photographs of the fabricated white LEDs. $\mathbf{c}$ Experimental spectra of the white LED without the sample $(x=0)$, white LED with the sample $(x=1)$ and theoretical spectrum $x=0.9$. $\mathbf{d}$ The $R_{a}$ index plot per $x$ for different theoretical spectra, $\operatorname{THEOR}(\lambda)=\operatorname{LED} 1(\lambda)+x \times \operatorname{DIFF}(\lambda)$ with a maximum at $x=0.9$ and theoretical maximum $R_{\mathrm{a}}$ (theoretical max) $=95.7$

experimental spectrum of LED1. The different theoretical spectrum $\operatorname{THEOR}(\lambda)$ can therefore be presented as the sum of spectrum $\operatorname{LED}(\lambda)$ without sample and different additions of $\operatorname{DIFF}(\lambda)$ with scaling coefficient $x$ : THEOR $(\lambda)$ $=\operatorname{LED} 1(\lambda)+x \times \operatorname{DIFF}(\lambda)$. It should be noted that $x=0$ means a pure LED1 $(\lambda)$ spectrum and $x=1$ is a pure LED2 $(\lambda)$ spectrum. Several theoretical spectra were obtained by varying $x$ in the range of $x=0-2$ with a step size of 0.1 . The $R_{\mathrm{a}}$ index of each spectrum was calculated, and the dependence of $R_{\mathrm{a}}$ on $x$ was plotted (Fig. 4d). The maximum in the $R_{\mathrm{a}}(x)$ function is $x=0.9$ with $R_{\mathrm{a}}(\max )=95.7$. Therefore, the $R_{\mathrm{a}}$ of a LED with cyan phosphor NKLSO: $\mathrm{Eu}^{2+}$ can be raised to 95.7, and the fabricated LED2 with $R_{\mathrm{a}}=95.2$ is close to this maximum (Fig. $4 \mathrm{~d}$ ). The spectrum with this theoretical maximum was calculated using the equation of $\operatorname{THEOR}(\lambda)$ and $x=0.9$, and it also showed similarity with the spectrum of LED2 (Fig. 4c), which indicates that the theoretical prediction is reasonable. However, the simulation of optimal peak emission and optimal emission band width is far from reality.
Accordingly, by determining the optimal peak emission wavelength in combination with the optimal emission band width of a special cyan-emitting phosphor, the idea mentioned above can be used in future work, especially for similar compounds, by revealing the mechanism that improves the $R_{\mathrm{a}}$ value.

In summary, we have identified a novel ultra-narrowband cyan-emitting NKLSO:Eu ${ }^{2+}$ phosphor with $\lambda_{\mathrm{em}}=$ $486 \mathrm{~nm}$, an FWHM of only $20.7 \mathrm{~nm}$ and a Stokes shift of $1069 \mathrm{~cm}^{-1}$. The unprecedented ultra-narrow-band cyan emission and extremely small Stokes shift are attributed to the highly condensed, rigid framework and high cubic symmetry sites for the activator $\left(\mathrm{Eu}^{2+}\right)$. The cyan-emitting phosphor also shows good thermal quenching properties with an integrated emission intensity of $93 \%$ at $150{ }^{\circ} \mathrm{C}$, demonstrating that NKLSO: $\mathrm{Eu}^{2+}$ is a promising phosphor for enhancing the color-rendering index of pc-LEDs in full-spectrum lighting applications. By combining this newly discovered cyan phosphor NKLSO:8\% $\mathrm{Eu}^{2+}$, the commercial yellow phosphor YAG:Ce and the 
commercial red phosphor KSF: $\mathrm{Mn}^{4+}$ with a blue InGaN LED chip, we fabricated a warm white LED with an $R_{\mathrm{a}}$ of 95.2. Compared with that of the white LED without the NKLSO: $\mathrm{Eu}^{2+}$, the $R_{\mathrm{a}}$ has been improved significantly, indicating that the phosphor can compensate for the cyan gap and demonstrates great potential for high-CRI white LED devices.

\section{Materials and methods}

\section{Synthesis}

The designed samples of NKLSO: $x \mathrm{Eu}^{2+}(x=0-15 \%)$ were synthesized using the traditional solid-state reaction. Stoichiometric amounts of $\mathrm{Na}_{2} \mathrm{CO}_{3}$ (A.R., Aladdin), $\mathrm{K}_{2} \mathrm{CO}_{3}$ (A.R., Aladdin), $\mathrm{Li}_{2} \mathrm{CO}_{3}$ (A.R., Aladdin), $\mathrm{SiO}_{2}$ (A. R., Aladdin), and $\mathrm{Eu}_{2} \mathrm{O}_{3}$ (99.99\%, Aladdin) were homogeneously mixed and ground with ethanol for half an hour. The mixtures were first sintered at $550^{\circ} \mathrm{C}$ for $5 \mathrm{~h}$ in air and then sintered three times at $750^{\circ} \mathrm{C}$ for $4 \mathrm{~h}$ under a reducing atmosphere of $\mathrm{N}_{2}-\mathrm{H}_{2}(10 \%)$ in a tube furnace. The obtained samples were cooled to RT and were reground into fine powders for further characterization.

\section{Characterization}

The powder X-ray diffraction (XRD) data of NKLSO: $x \mathrm{Eu}^{2+}$ for Rietveld analysis were collected at RT by a Bruker D8 ADVANCE powder diffractometer, operating at $40 \mathrm{kV}$ and $40 \mathrm{~mA}$ with monochromatized $\mathrm{Cu} \mathrm{K \alpha}$ radiation $(\lambda=1.5406 \AA)$. Rietveld refinement was performed using TOPAS 4.2. A ${ }^{7} \mathrm{Li}$ solid-state NMR spectrum was obtained on a JNM-ECZ600R instrument at 15 $\mathrm{kHz}$, and the external reference was $\mathrm{LiCl}$. The morphology of the powder sample was observed by SEM (SEM, JEOL JSM-6510). The elemental composition and elemental mapping were obtained using energy dispersive EDS that was attached to the SEM. The diffuse reflectance spectra at RT were measured on a Hitachi UH4150 ultraviolet-visible-near infrared spectrophotometer with white $\mathrm{BaSO}_{4}$ for calibration. The PL and PLE spectra at RT were recorded by an Edinburgh FLS920 fluorescence spectrophotometer with a Xe900 lamp as the excitation source. The internal/external quantum efficiency values were measured using the integrating sphere on the same FLS920 instrument, and white $\mathrm{BaSO}_{4}$ powder was used as a reference to measure the absorption. The luminescence decay curves were measured by an FLS920 instrument using an nF900 flash lamp as the excitation source. The low-temperature $(80 \mathrm{~K})$ measurements were performed using the FLS920 with an Oxford Instrument, and the sample was cooled by liquid nitrogen. The temperaturedependent spectra were obtained by a Hitachi F-4600 fluorescence spectrophotometer with a heating apparatus as the heating source and a $150 \mathrm{~W}$ Xe lamp as the excitation source.

\section{WLED fabrication}

White LEDs were fabricated with the cyan phosphor NKLSO:8\% $\mathrm{Eu}^{2+}$, the commercial yellow phosphor YAG: $\mathrm{Ce}$, the commercial red phosphor KSF: $\mathrm{Mn}^{4+}$ and a blue LED InGaN chip $(\lambda=455 \mathrm{~nm})$. The phosphors were thoroughly mixed with epoxy resin, and the obtained phosphor-epoxy resin mixture was coated on the LED chips. The photoelectric properties, including the emission spectra, CCT, color-rendering index (CRI, $R_{\mathrm{a}}$ ), luminous efficacy, and CIE color coordinates of the LEDs, were measured by using an integrating sphere spectroradiometer system (ATA-1000, Ever fine).

\section{Acknowledgements}

The present work was supported by the National Natural Science Foundations of China (Grant Nos. 51722202, 51572023 and 91622125), Natural Science Foundations of Beijing (2172036), Fundamental Research Funds for the Central Universities (FRF-TP-18-002C1), and the Guangdong Provincial Science \& Technology Project (No. 2018A050506004). M.S.M. is grateful for the support from the Russian Foundation for Basic Research (Grant No. 17-52-53031).

\section{Author details}

${ }^{1}$ The Beijing Municipal Key Laboratory of New Energy Materials and Technologies, School of Materials Sciences and Engineering, University of Science and Technology Beijing, 100083 Beijing, China. ${ }^{2}$ Laboratory of Crystal Physics, Kirensky Institute of Physics, Federal Research Center KSC SB RAS, Krasnoyarsk, 660036, Russia. ${ }^{3}$ Siberian Federal University, Krasnoyarsk 660041, Russia. ${ }^{4}$ Department of Physics, Far Eastern State Transport University, Khabarovsk 680021, Russia. ${ }^{5}$ State Key Laboratory of Luminescent Materials and Devices and Guangdong Provincial Key Laboratory of Fiber Laser Materials and Applied Techniques, South China University of Technology, 510641

Guangzhou, China

\section{Author contributions}

Z.G.X. initiated and guided the research. M.Z. and Z.G.X. discussed and wrote the manuscript, and Q.L.L. and Q.Y.Z. commented on it. M.Z. and H.X.L. performed the experiments. M.S.M. performed Rietveld refinement of the power X-ray diffraction results. Y.Y.Z. and Q.Y.Z. fabricated the WLEDs and analyzed the emission spectra of the white LEDs.

\section{Conflict of interest}

The authors declare that they have no conflict of interest.

Supplementary information is available for this paper at https://doi.org/ 10.1038/s41377-019-0148-8.

Received: 7 August 2018 Revised: 25 March 2019 Accepted: 26 March 2019 Published online: 10 April 2019

\footnotetext{
References

1. Pust, P., Schmidt, P. J. \& Schnick, W. A revolution in lighting. Nat. Mater. 14 454-458 (2015).

2. Pimputkar, S. et al. Prospects for LED lighting. Nat. Photon. 3, 180-182 (2009).

3. Schubert, E. F. \& Kim, J. K. Solid-state light sources getting smart. Science $\mathbf{3 0 8}$ 1274-1278 (2005).

4. Xia, Z. G. \& Meijerink, A. Ce ${ }^{3+}$-Doped garnet phosphors: composition modification, luminescence properties and applications. Chem. Soc. Rev. 46, 275-299 (2017).

5. Xia, Z. G. \& Liu, Q. L. Progress in discovery and structural design of color conversion phosphors for LEDs. Prog. Mater. Sci. 84, 59-117 (2016).

6. Meyer, J. \& Tappe, F. Photoluminescent materials for solid-state lighting: state of the art and future challenges. Adv. Optic. Mat. 3, 424-430 (2015).
} 
7. Huang, L. et al. HF-free hydrothermal route for synthesis of highly efficient narrow-band red emitting phosphor $\mathrm{K}_{2} \mathrm{Si}_{1-x} \mathrm{~F}_{6} \times \mathrm{Mn}^{4+}$ for warm white lightemitting diodes. Chem. Mater. 28, 1495-1502 (2016).

8. Xie, R. J. et al. A simple, efficient synthetic route to $\mathrm{Sr}_{2} \mathrm{Si}_{5} \mathrm{~N}_{8}: \mathrm{Eu}^{2+}$-based red phosphors for white light-emitting diodes. Chem. Mater. 18, 5578-5583 (2006).

9. Piao, X. Q. et al. Preparation of $\mathrm{CaAlSiN}_{3}: \mathrm{Eu}^{2+}$ phosphors by the selfpropagating high-temperature synthesis and their luminescent properties. Chem. Mater. 19, 4592-4599 (2007).

10. Pust, $P$. et al. Narrow-band red-emitting $\operatorname{Sr}\left[\mathrm{LiAl}_{3} \mathrm{~N}_{4}\right]: \mathrm{Eu}^{2+}$ as a next-generation LED-phosphor material. Nat. Mater. 13, 891-896 (2014).

11. Wang, $\mathrm{L}$. et al. $\mathrm{Ca}_{1-x} \mathrm{~L} \mathrm{i}_{x} \mathrm{Al}_{1-x} \mathrm{Si}_{1+x} \mathrm{~N}_{3}: \mathrm{Eu}^{2+}$ solid solutions as broadband, colortunable and thermally robust red phosphors for superior color rendition white light-emitting diodes. Light Sci. Appl. 5, e16155 (2016).

12. Dai, P. P. et al. A single Eu ${ }^{2+}$-activated high-color-rendering oxychloride whitelight phosphor for white-light-emitting diodes. Light Sci. Appl. 5, e16024 (2016).

13. Strobel, $P$. et al. Luminescence of an oxonitridoberyllate: a study of narrowband cyan-emitting $\mathrm{Sr}_{[}\left[\mathrm{Be}_{6} \mathrm{ON}_{4}\right]: \mathrm{Eu}^{2+}$. Chem. Mater. 30, 3122-3130 (2018).

14. Ohno, Y. Color rendering and luminous efficacy of white LED spectra. In Proc. the SPIE 49th Annual Meeting 5530, 88-98 (SPIE, Denver, Colorado, 2004).

15. Phillips, J. M. et al. Research challenges to ultra-efficient inorganic solid-state lighting. Laser Phot. Rev. 1, 307-333 (2007)

16. Li, Y. Q. et al. Luminescence properties of $\mathrm{Eu}^{2+}$-activated alkaline-earth siliconoxynitride $\mathrm{MSi}_{2} \mathrm{O}_{2-\delta} \mathrm{N}_{2+2 / 3 \delta}(\mathrm{M}=\mathrm{Ca}, \mathrm{Sr}, \mathrm{Ba})$ : a promising class of novel LED conversion phosphors. Chem. Mater. 17, 3242-3248 (2005).

17. Zhao, M. et al. Discovery of new narrow-band phosphors with the $\mathrm{UCr}_{4} \mathrm{C}_{4}$ related type structure by alkali cation effect. Adv. Optic. Mat. 7, 1801631 (2019).

18. Xia, Z. G. et al. Chemical unit cosubstitution and tuning of photoluminescence in the $\mathrm{Ca}_{2}\left(\mathrm{Al}_{1-x} \mathrm{Mg}_{x}\right)\left(\mathrm{Al}_{1-x} \mathrm{Si}_{1+x}\right) \mathrm{O}_{7}: \mathrm{Eu}^{2+}$ phosphor. J. Am. Chem. Soc. 137, 12494-12497 (2015).

19. Strobel, $P$. et al. Ultra-narrow-band blue-emitting oxoberyllates $A E L i_{2}\left[\mathrm{Be}_{4} \mathrm{O}_{6}\right]$ : $\mathrm{Eu}^{2+}(\mathrm{AE}=\mathrm{Sr}, \mathrm{Ba})$ paving the way to efficient RGB pc-LEDs. Angew. Chem. Int. Ed. 130, 8875-8879 (2018)
20. Strobel, P. et al. Narrow-band green emitting nitridolithoalumosilicate $\mathrm{Ba}$ $\left[\mathrm{Li}_{2}\left(\mathrm{Al}_{2} \mathrm{Si}_{2}\right) \mathrm{N}_{6}\right]: \mathrm{Eu}^{2+}$ with framework topology whj for LED/LCD-backlighting applications. Chem. Mater. 27, 6109-6115 (2015).

21. Strobel, $\mathrm{P}$. et al. $\mathrm{Sr}\left[\mathrm{BeSi}_{2} \mathrm{~N}_{4}\right]: \mathrm{Eu}^{2+} / \mathrm{Ce}^{3+}$ and $\mathrm{Eu}\left[\mathrm{BeSi}_{2} \mathrm{~N}_{4}\right]$ : nontypical luminescence in highly condensed nitridoberyllosilicates. Chem.-Eur. J 24, 7243-7249 (2018).

22. Liao, H. X. et al. Learning from a mineral structure toward an ultra-narrowband blue-emitting silicate phosphor $\mathrm{RbNa}_{3}\left(\mathrm{Li}_{3} \mathrm{SiO}_{4}\right)_{4}: \mathrm{Eu}^{2+}$. Angew. Chem. Int. Ed. 130, 11902-11905 (2018)

23. Zhao, M. et al. Next-generation narrow-band green-emitting $\mathrm{RbLi}\left(\mathrm{Li}_{3} \mathrm{SiO}_{4}\right)_{2}$ $\mathrm{Eu}^{2+}$ phosphor for backlight display application. Adv. Mat. 30, 1802489 (2018).

24. Dutzler, D. et al. Alkali lithosilicates: renaissance of a reputable substance class with surprising luminescence properties. Angew. Chem. Int. Ed. 57, 13676-13680 (2018)

25. Wang, S. X. et al. Crystal field splitting of $4 \mathrm{f}^{\mathrm{n}-1} 5 \mathrm{~d}$-levels of $\mathrm{Ce}^{3+}$ and $\mathrm{Eu}^{2+}$ in nitride compounds. J. Lumin. 194, 461-466 (2018).

26. Hoffmann, J., Brandes, R. \& Hoppe, R. Neue silicate mit "stuffed pyrgoms":

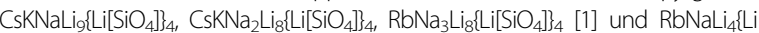
$\left[\mathrm{SiO}_{4}\right]_{2}$ [2]. Z. Anorg. Allg. Chem. 620, 1495-1508 (1994).

27. Blasse, G. \& Grabmaier, B. C. Luminescent materials (Springer, Berlin Heidelberg, 1994).

28. Van Uitert, L. G. An empirical relation fitting the position in energy of the lower d-band edge for $\mathrm{Eu}^{2+} \mathrm{OR} \mathrm{Ce}^{3+}$ in various compounds. J. Lumin. 29, 1-9 (1984).

29. Kim, Y. H. et al. A zero-thermal-quenching phosphor. Nat. Mater. 16, 543-550 (2017).

30. Blasse, G. Thermal quenching of characteristic fluorescence. J. Chem. Phys. $\mathbf{5 1}$ 3529-3530 (1969).

31. Denault, K. A. et al. Average and local structure, Debye temperature, and structural rigidity in some oxide compounds related to phosphor hosts. ACS Appl. Mater. Interfaces 7, 7264-7272 (2015). 